\title{
Use of Yoked and Differential Prisms to Improve Posture
}

\author{
Jonathan Shapiro, BSc(Hons), \\ FCOptom, FAAO, FEAOO \\ Optometrist, \\ Former Senior Lecturer \\ Department of Optometry \\ Hadassah Academic College, \\ Jerusalem
}

\begin{abstract}
This paper presents cases of postural changes, and the use of prisms to relieve symptoms, caused by A and V syndromes (with a suggestion as to their cause); asymmetry of the position of the mastoid process; facial asymmetry resulting from a disparate growth rate of the skull; slack-jaw; Duane's syndrome and $6^{\text {th }}$ nerve palsy, as well as stooping in elderly patients. Although Yoked and Differential prisms are used for the treatment and alleviation of symptoms experienced by patients who have suffered mild traumatic brain injury, this is outside the scope of this paper. The methods for examining patients and prescribing prisms are described.
\end{abstract}

\section{KEY WORDS:}

Yoked and Differential Prisms, A and V Syndromes, Posture, Falling, Spatial Perception and Localization.

A Differential prism changes the spatial localization of an image in one eye. A Yoked prism is a pair of prisms of equal power and direction that change the spatial localization of both eyes equally. Differential prisms are traditionally prescribed to alleviate the symptoms of binocular vision stress, while Yoked prisms are used to re-locate the posture. A prescription can combine both types of prisms. Secondary effects of prescribing prisms such as spatial distortion, expansion and compression will be discussed later in the paper.

The effects of Yoked and Differential prisms on posture are well established in the literature..$^{-4}$ Kaplan $^{1}$ laid the foundation for the use of vertical Yoked prisms in optometric care to alleviate a variety of symptoms associated with the perception of visual space. Kaplan's focus was on accommodation and convergence. Research has shown that vertical Yoked prisms have minor effects on accommodation and binocular vision, at least during short-term wear, in young adults with normal binocular vision. ${ }^{5,6}$ This paper is not concerned with the treatment of binocular vision anomalies, but rather focuses on the treatment and prevention of damage and discomfort caused by postural adaption.

This paper continues the work of previous authors and considers the use of prisms, both Yoked and Differential, in routine practice to manipulate the posture. Example cases presented here show the use of prisms for various conditions.

Although Yoked prisms have been widely used to improve functionality in cases of traumatic brain injury (TBI) and other neurological conditions, these applications are not considered in this paper ${ }^{3,7-10}$ However, since many of the concepts presented in this paper are influenced by the research and experience gained in treating TBI, it is valid to include that information here.

Egocentric Localization (EL) has been defined as the awareness of the spatial positions of objects in the immediate environment relative to one's body. The absence of this state is termed Abnormal Egocentric Localization (AEL), ${ }^{11,12}$ or Visual Midline Shift Syndrome (VMSS). The former term is preferred recently, as it is uncertain whether the "shift" is purely visual. While it can change, for example, before and after vestibular activity, it can 
respond very well to Yoked prisms; therefore, it is likely a mixture of both visual and vestibular components. ${ }^{11,12}$ Padula and co-workers ${ }^{11,12}$ noted that, in normal conditions, the visual midline is determined and stabilized during early development of the central nervous system. This establishes the proper alignment of the body for the maintenance of balance during motion. ${ }^{13}$ By integrating information from the visual system with the proprioceptive base of support and vestibular input, postural alignment maintains balance. The combination creates a stable visual midline.

It has been suggested that the spatial perception dominant eye may contribute to this balance. This notion is supported by findings from Velay et al., ${ }^{14}$ who showed that proprioceptive signals arising from both eyes are involved in egocentric visual localization. Proprioceptive dominance was observed, but of the dominant eye.

If the dominant eye is degraded, for example by monocular ARMD or cataract, the visual midline may be disrupted. The non-dominant spatial perception eye now has significantly better vision than the eye previously used to determine spatial localization. This paper refers to this condition as Displaced Spatial Perception. Yoked lateral prisms may be prescribed to relocate the perceived image close to the habitual spatial localization.

The use of Yoked prisms creates the condition of a new visuo-motor and motor-sensory response that results in behavioural changes. ${ }^{1,11-13}$ Yoked prisms cause changes in posture, since they affect the perception of peripheral visual space. The visual system can affect how a person adjusts their head position to achieve comfortable extra-ocularmuscle (EOM) balance, in addition to proper visual midline perception and proper egocentric localization.

The use of Yoked and Differential prisms does not treat the underlying condition. Instead, the prisms in the prescription produce a compensatory mechanism. This alleviates the need for postural adjustment that the patient may have adopted to reduce the symptoms of ocular discomfort.

The maintenance of posture is achieved by a combination of many factors. To maintain balance, the input from the visual system, vestibular system, and proprioception pressure receptors of the neck and the feet on the ground should all be in synchronization. The visual system is the dominant system and can override the other two systems. This can be demonstrated by having a subject walk through a chequered-wall tunnel. When the chequered pattern on the walls of the tunnel rotates, the subject falls in the direction of the rotation, although the other vestibular and proprioception systems are functioning normally. ${ }^{15}$

The effect of posture on the visual system and the effect of the visual system on posture are connected. It is uncertain which is the dominant partner or if the interaction is equally balanced.

We can conjecture that, by improving the posture, through the re-aligning and relaxation of tension on the EOM, we can improve the maintenance of the peripheral visual space needed for balance. The further advantage of reducing possible long-term damage to the spinal cartilage and supporting muscles should also be considered.

POSSIBLE RELATION BETWEEN A AND V SYNDROMES AND ORBITAL ROTATION

$A$ and $V$ syndromes are noted when the maintenance of single binocular vision, without tension or with reduced tension on the extra-ocular muscles, only occurs with an upward (A) or downward gaze (V), rather than in the primary position. On looking in the opposite direction of the position of comfort, there is a tendency for the eyes to break into an exophoria that results in difficulty in maintaining single binocular vision.

In cases of A syndrome, there is a tendency to hold the head down to elevate the eyes to the top of the A, where the binocular muscle balance is most comfortable. This head position is reversed in cases of V syndrome. It is suggested that a further reason to drop the head, and raise the eyes into the orbit, may be to create a form of dampening of the micro-nystagmic oscillations caused by stress on the EOM when in the primary position.

Quaid and Hamilton-Wright, ${ }^{16}$ comparing the Hess examination to the Parks 3-step test, noted that, in a case of esodeviation, there will be a different pattern, and the movements will be reversed.

The binocular vision anomalies encountered with A and V syndromes resulting from tropias (commonly $15^{\Delta}$ difference between the two positions of gaze) are not considered here. These are normally caused by problems of nerve supply, malpositioned muscle insertion or muscle under-action. 
The cause of non-pathological A and V syndromes has not been fully ascertained. Urrets-Zavalía and co-workers ${ }^{17,18}$ introduced an interesting concept. Working in Argentina in the 1950s, they noted that the pure native population (termed Mongoloid) had upturned palpebral apertures, while the non-native population (pure European, termed Anti-Mongoloid) tended to have straight or downturned palpebral apertures. They suggested that the position of the orbital rotation influenced the location of the EOM insertion. It is suggested here that this may be the cause of A and V syndromes. Urrets-Zavalía and co-workers considered that the rotational position of the orbits is influenced by the relative development of the zygomatic bone (cheekbone). If the zygomatic bone develops as expected, the orbits of the eyes are in the level orientation and the insertion points of the lateral and medial recti lie on the same plane. Hypo-plasial development of the zygomatic bone will result in the orbit not fully rotated, while hyper-plasial development will create an over-turned orbit. This will be noted by the appearance of palpebral apertures. In their words, "(i)t thus appeared that the aforementioned disturbances of the ocular muscles are in some way related to the accompanying disorders of the facial scaffolding."

When the palpebral apertures appear to be horizontal, assuming the orbits follow the structure of the palpebral position, the vergence movements maintain their relative position and move smoothly. There is no cyclic tension on the EOM during vergence or convergence. The head maintains a straight posture.

When there is hypo-plasial development of the zygomatic bone, the orbital rotational development is not completed. The medial recti lie on a plane higher than the lateral recti. This leads to a V syndrome and the chin is raised.

When there is hyper-plasial development of the zygomatic bone, there is an over-turned orbital structure. The lateral recti are positioned higher than the medial recti and an A syndrome occurs and the chin is lowered.

An asymmetrical development of the zygomatic bone results in one orbit being straight while the other orbit is under- or over-turned. There will also be vertical facial asymmetry. As an aside, if the upturned orbit is the dominant eye, there is a tendency to head-tilt on tracking to align the medial and lateral recti (Fig. 1).

Figure 1: Relative position of the orbits. (a) Palpebral aperture straight. (b) Bilateral Upturn. (c) Bilateral downturn. Asymmetry: (d) Right orbit upturn, left orbit straight. (e) Right orbit upturn, left orbit straight and elevated.

(a)

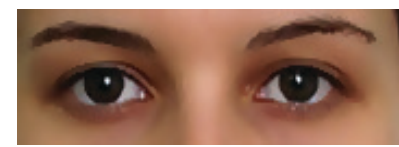

(d)

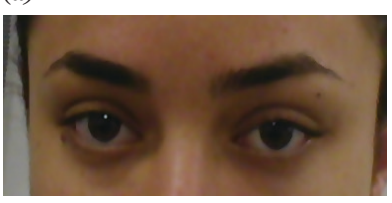

(b)

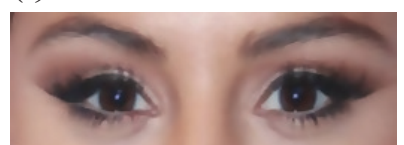

(e) (c)
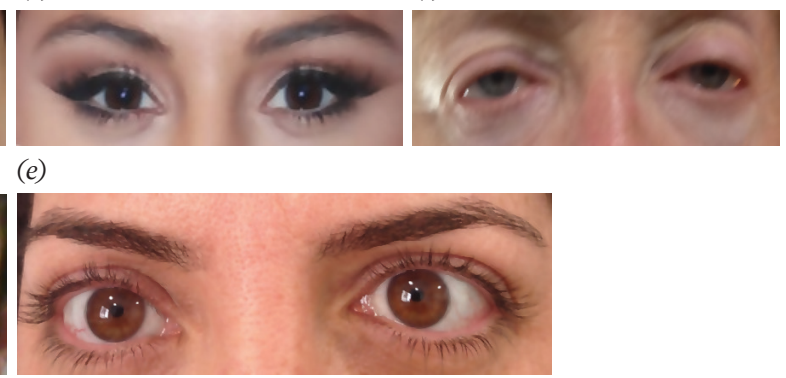

The raised or lowered chin affects the posture. Adaptions are made in the spine to maintain the centre of gravity.

\section{METHOD FOR MONITORING THE RELATIVE POSITION OF THE EYES DURING VERGENCES}

Equipment for monitoring the relative positions of the eyes during vergences is not usually available in clinical work. The following system has been designed for monitoring the effect of vertical imbalances and guiding the prescription of Yoked prisms.

Variations in the relative vertical position of a perceived object can be demonstrated, and noted clinically, by carefully measuring the vertical balance as the head moves slowly left and right, while fixating on a central target. The relative position is examined with the head held in the primary position, repeated with the chin depressed, and again with the chin raised. 
Figure 2: (a) Targets used to determine the relative position of the eyes on tracking. The nonius lines are oriented horizontally and vertically. (b) The Parallel-Testing Infinity Balance (PTIB). The whole target is seen by both eyes simultaneously, except for the squares and attached red line, seen by each eye separately, which are shaded for explanation.

(a)

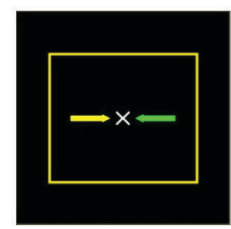

(b)

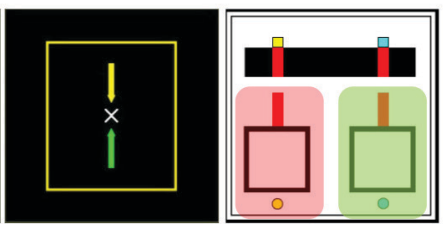

The examination uses different targets (Fig. 2). The first target has two nonius lines positioned along the horizontal, polarized for each eye. The colours chosen for the arrows are close to each other on the spectrum to avoid spatial displacement from transverse chromatic aberration (TCA). Peripheral locking is maintained by the use of a yellow square. The positions of the nonius lines in relation to the $\mathrm{X}$, and to each other, are compared as the head moves laterally while fixating on the X. The head of the patient is held in the primary position by the examiner to prevent head-tilting. By holding the head during the examination, the examiner can control the speed of movement. The head is moved slowly left and right. If no variations in the vertical positioning of the images are found, the positioning of the lateral and medial recti can be assumed to be level. A major difference in the relative vertical height of the two eyes, at the edge of the vergence movements, indicates an imbalance. If the lateral and medial recti muscles are not aligned, as the head moves to one side, one line will rise or drop. On moving in the opposite direction, the opposing line will move. This action is repeated with the chin depressed and raised. If there is a reduction in the amount of vertical movement during vergences, comparing the primary position to when the chin is depressed or raised, then Yoked vertical prisms are introduced. The power of the Yoked prisms prescribed is determined by the amount needed to reduce vertical movement to a minimum, when the head is held in the primary position. After the Yoked prisms are introduced, the test is repeated with the nonius line positioned vertically, to determine if there is any disruption to the horizontal balance.

The trial frame should be positioned carefully to avoid inducing Yoked prisms in high prescriptions. If the prescription is low, the examination is better performed without glasses. If there are oblique cylinders in the prescription, the examination is compromised by the spatial dislocation caused by lateral movements.

The third target, the Parallel-Testing Infinity Balance (PTIB), ${ }^{19,20}$ has multiple uses for examining the relationship of the visual space, as seen by each eye, relative to the binocular visual space.

The top black bar, with 2 red lines, is seen binocularly. The squares, with attached red lines, are seen separately by each eye. In this test, the PTIB will show the vertical and horizontal misalignment of the images perceived by each eye during motion. This is noted by the patient as changes in the vertical alignment of the two squares, and/or the relative horizontal positioning of the red nonius lines on the squares with red lines in the black bar. It will also show any cyclo-rotation or image-size variation. The examination will also highlight possible involvement of the oblique muscles.

The head is held in the primary position. If necessary, Differential prisms are used to balance the vertical and horizontal imbalances before the head is moved. After the position of the squares is balanced, the head is moved laterally and vertically, as described before. The patient reports if the squares remain on the same level, if they cyclo-rotate away from the original format, if they change size, or if the red line on the square dislocates from the red line in the black bar above. The information allows the practitioner to infer the actions of the EOM. Whether there is an over- or under-action of a specific muscle is less important than the relation of the paired muscles during tracking. A cyclic rotation, as exhibited on the squares of the PTIB, may be modified when the head is held in a specific position.

Positioning of the orbits may also be influenced by under-development or over-development of the nasal projection, causing in-turning or out-turning of the orbits (Fig. 3).

Figure 3: (a) Over-development, (b) under-development, and (c) asymmetrical development of the nasal projection.

(a)

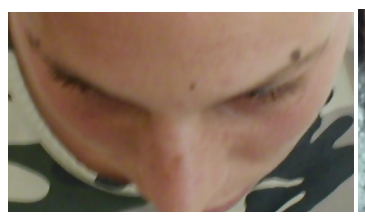

(b)

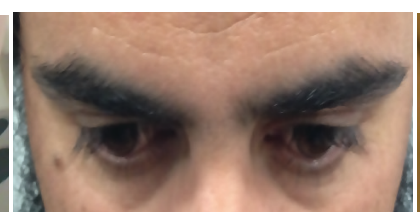

(b)

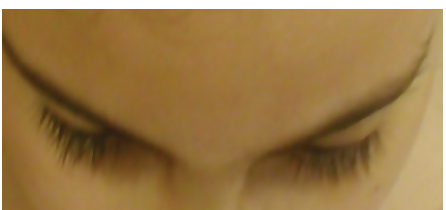


EFFECTS OF THE A AND V SYNDROMES ON POSTURE

The severity of vergence and accommodative imbalances, the flexibility of the system with alteration of the head position, and the introduction of vertical prism have been debated. Quaid and Simpson ${ }^{21}$ showed a correlation with reading efficiency using infra-red tracking. In cases of excess forward or backward head-tilting, training was required to improve the flexibility of the vergence / accommodative system. The relaxation of stress on the neck muscles reduces the imbalance and, in time, a person will read with improved comfort (Fig. 4). Treating the flexibility of the vergence/ accommodative system by training may be adequate to alleviate the problems and may be used instead of, or in conjunction with, Yoked prisms. Other research concluded that the effect on the horizontal phoria of the inclusion of vertical prisms is of no consequence, although A and V syndromes were not considered in that report. ${ }^{6}$

A patient exhibiting an A syndrome may lower their head by dropping their chin, thereby pushing the eye into the superior orbit. On dropping the chin, the centre of gravity of the body is moved forward. To compensate for this change, the back is arched inwards. This is reversed in a V syndrome. By incorporating a Yoked prism in the prescription, the head position is adapted, relaxing the stress on the posture, and allowing easier tracking.

Figure 4: (a) A syndrome without and with a Yoked down prism correction. (b) Arched back treated with a Yoked down prism.

(a)

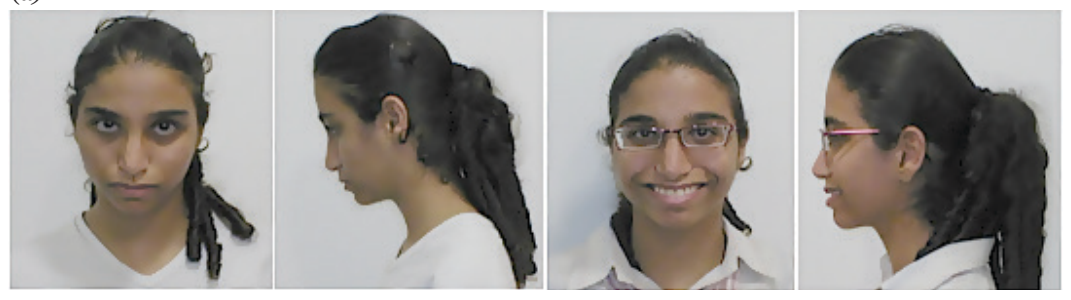

(b)

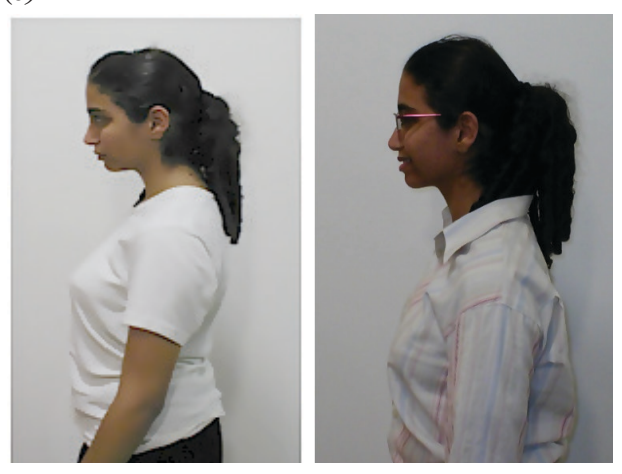

ASYMMETRY OF THE POSITION OF THE MASTOID PROCESS

Vertical facial asymmetry (VFA), due to either differential genetic growth of the skull or shortening of the sterno-cleidomastoid muscles (SCM), causes deformation of the skull and can also influence head-tilt. ${ }^{22,23}$ The mastoid process anchors the superior tendon of the SCM. The SCM plays a significant role in controlling the postural position of the head.

The SCM, trapezius and sub-occipital muscles begin to function at about 3 months after birth, which is about a month before the development of convergence, divergence and fusion. If there is a head-tilt of the positioning of the skull due to an asymmetry or imbalance of these muscles, it is possible that the vergences will not develop to their full potential. The relative location of the eyes may induce cyclo-rotation. Major and co-workers ${ }^{24}$ noted a high rate of oculo-motor failure in children who had been wheelchair-bound from a young age.

The positioning of the right and left mastoid processes may not be symmetrical. An anterior-posterior asymmetry will cause tension on the y-axis position of the head. In Fig. 5, the right mastoid process is situated further back than the left mastoid process. The head is turned on the y-axis to the right shoulder. The patient has various compensatory mechanisms available to maintain the positioning of the body and head. This will depend on the actions required. Walking requires the symmetrical positioning of the hips and shoulders. To achieve this, there will be a vergence movement to the left. In reading and computer use, the patient will keep the head straight and instead use a y-axis movement of the 
shoulders and hips. Monotonic walking on a treadmill while watching a TV screen placed directly in front can lead to disruption of the shoulder and lower back muscles. Maintaining the head-turn causes the right shoulder to move forward, which is then compensated for by twisting the lower spine in the opposite direction. Stressing the SCM to keep the head and spine straight can lead to neck ache. Patients with this structure exhibit seating preferences for certain positions such as watching TV, theatre, lecture rooms, positioning the computer on the desk, etc. If seated in an opposing position, they will twist themselves, leading to musculature stress. Horizontal Yoked prisms can be used if the turned head position is maintained, and the constant vergence disturbs normal tracking. It is worth noting that measuring the mono p.d. for multi-focals should be performed in the natural position, and not with the head straightened.

Figure 5: Anterior-posterior asymmetry of the position of the mastoid process. The head is turned to the right.

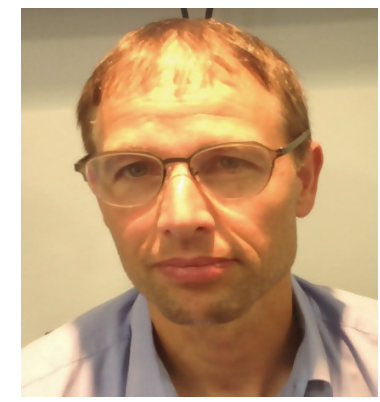

SHORTENING OF THE SCM

Another form of asymmetry is caused by malformation of the skull. One of the causes of congenital torticollis may be positioning of the skull in a cramped position in the womb, especially if the head was positioned superiorly. The distortion to the skull is usually on the right side, although it is found occasionally on the left side. Since the distortion usually occurs on the right side, it is postulated that the displaced lateral position of the liver, which presses on the womb, may be a contributing factor when the head is superiorly positioned. One of a pair of identical twins may exhibit the distortion, suggesting there is no genetic involvement (Fig. 6).

Figure 6: (a) Identical male twins. The sibling on the right has an asymmetrical shortening of the right SCM muscle. (b) The side of the face with a shorter, thicker SCM prevents normal growth of the skull. That side is smaller and more narrow than the unaffected side. The ear is located lower and the head tilts to that direction. Usually the aberration is on the right side (left), although it may occur on the left side (right).

(a)

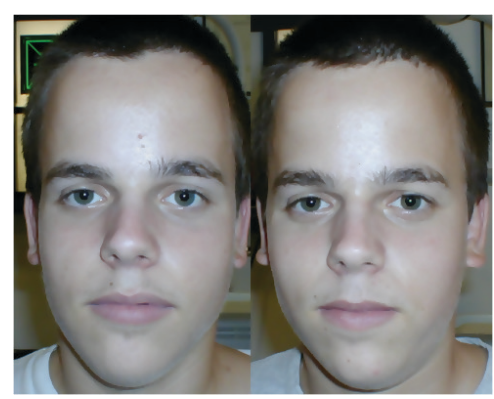

(b)

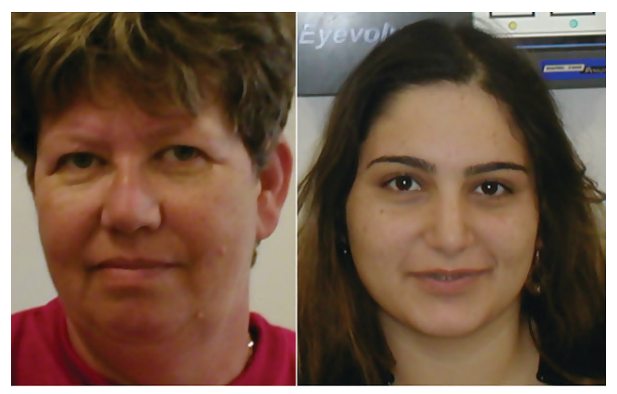

The SCM is shorter, thicker and less flexible on one side, usually the right side. It is difficult to straighten the head. Moving the head on the $y$-axis is freely achieved in one direction, but limited in the opposite direction. The patient may adjust their posture to lessen the stress on that side. There is a greater difference in the vertical heights of the eyes than in the first form of asymmetry noted above. The shoulders are more rounded and thicker than normal, and are asymmetric. The spine shows a bend at the neck (Fig. 7). The palpebral apertures are often reduced. The head-tilt leads to cyclo-rotation of the eyes. Depending on the severity of the head-tilt, there may be poor binocular function, often with suppression in one eye. If the head is straightened, the binocular function improves, but the stress on the shortened thicker SCM muscle causes pain. 
The condition is treated with physiotherapy, including stretching of the affected muscle and tendons. A Differential prism, in conjunction with physiotherapy, is used to equalize the position of the perceived image in each eye.

Figure 7: (a) SCM shortening of the right side. The eyes are positioned on different levels. There is a minor cyclo-rotation of the eyes. The palpebral apertures are often reduced. (b) The shoulders develop with a different structure. (c) There may be a slight shift of the spine at the neck.

(a)

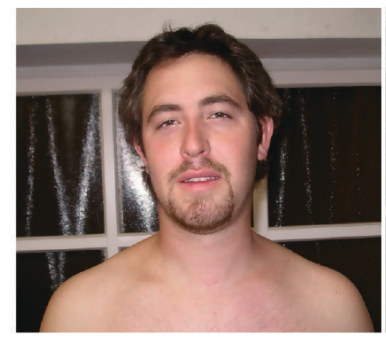

(b)

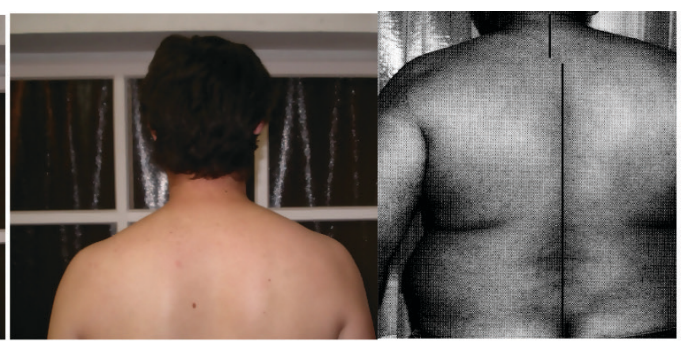

In Fig. 8 , the patient was treated by the initial introduction of $1^{\Delta}$ base down in front of the right eye, to be increased as necessary as treatment progressed. It is important to continue stretching the right SCM with aggressive physiotherapy. This use of the prism together with physiotherapy straightened the head and reduced the cyclo-rotation. The ability to track improved. If no physiotherapy is given, to lengthen the right SCM, the introduction of a prism causes a small compensatory bend at the level of the shoulders and lower back, and is detrimental to the patient.

Figure 8: (a) ) Right sided SCM shortening causes a head-tilt to the right shoulder. The eyes are on different levels. There is cyclo-rotation. There is a reduction in the ability to track and stereopsis is reduced. (b) Use of physiotherapy and differential vertical prisms straightened the head posture.

(a)

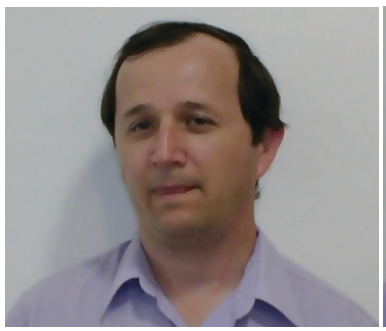

(b)

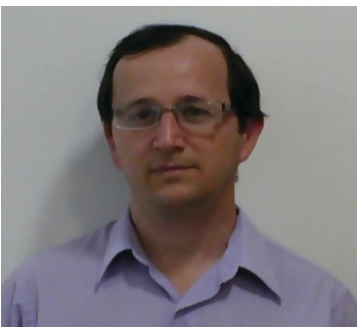

Figure 9 shows a simple form of physiotherapy that may be carried out at home. This therapy is performed for a few minutes daily until the required flexibility is attained. The head of the subject is held against the chest of the person stretching to protect the spine at the neck. The person carrying out the stretching uses one arm as contra-tension while the second arm applies tension. On reaching the last position of comfort, a small amount of extra tension is applied to lengthen the ligaments. The action is applied in both the $\mathrm{x}$ and $\mathrm{y}$ axes. It is worthwhile to stretch in both directions, although one direction is usually flexible.

Figure 9: Stretching to lengthen the ligaments of the sterno-cleido-mastoid. By balancing the tension of both SCM muscles, the head position can be held straight and the spine aligned.

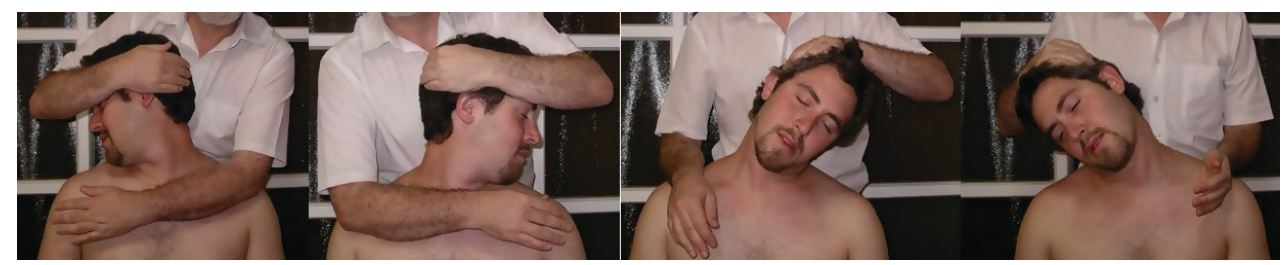


The patient in Fig. 10 suffered from epiphoria onto the right cheek. The patient exhibited both hypoplastic development of the right zygomatic bone and a shortened right SCM muscle. The head was positioned leaning to the right, with the outer canthus significantly lower than the inner canthus. This reversed the natural flow of the tears, and the flow was away from the drainage punctum. The outcome was epiphoria onto the right cheek. The introduction of a $1.5^{\Delta}$ base down prism in the right eye resulted in straightening of the head posture. The tear-flow direction was reversed and there was an immediate cessation of the epiphoria. The patient did experience stress on the right SCM, which was treated with physiotherapy and stretching of the neck muscles.

Figure 10: Use of vertical differential prism to alleviate tearing.

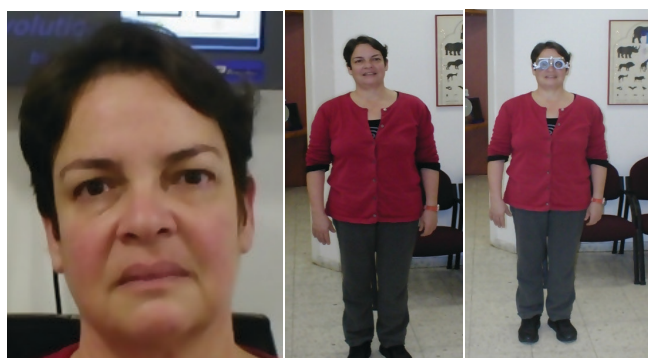

VERTICALLY DISPLACED MASTOID PROCESS, DUE TO GENETIC GROWTH DIFFERENCE, WITHOUT SCM INVOLVEMENT Patients may have equally developed SCM muscles, without the shortening and thickening as found with torticollis, but the positioning of the mastoid process is asymmetric. The head has free and equal movement in all directions, which is not found with torticollis. When the mastoid process on one side is positioned higher than that on the other side, the head tends to equalize the stress on the SCM by head-tilting. When this is combined with anterior-posterior skull developmental asymmetry, with the mastoid process being further back on one side of the skull, the shoulders tend to curve inwards. There is a tendency to crouch forwards. This results in the depth of breathing being reduced, as the lungs cannot expand fully. There is a cyclo-rotation of the eyes, creating problems in tracking. By the introduction of a Yoked base down prism, combined with a Differential prism base down in the right eye, the posture is improved (Fig. 11).

Figure 11: (a) Vertical and anterior-posterior asymmetric positioning of the mastoid process. This causes the right SCM muscle to pull the head to the right shoulder, and both shoulders to turn inward. (b) Yoked and Differential prisms re-align the head and the shoulders.

(a)

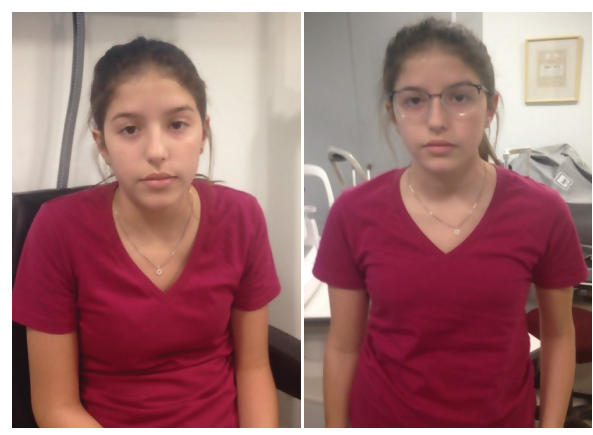

The patient in Fig. 12 presented with learning and social behavioural problems. His inability to read fluently, reduced progress in learning, clumsiness, and natural shyness made his social interaction and self-esteem in school problematic. He had taken to comfort-eating and had become overweight.

He exhibited a combination of vertical and anterior-posterior mastoid process asymmetry, combined with an A syndrome. He had a very minor ametropia.

Introducing $5^{\Delta}$ base down RE and 4 1/4 base down LE prisms reduced the head-tilt and straightened the posture. There was a marked improvement in his academic ability, clumsiness and social acceptability. There was a significant reduction in comfort-eating, as can be seen in his posture and weight a year later. Very little physiotherapy will be needed as the SCM muscles are not contracted. There were, however, repercussions. The final picture shows that there was an increase in the bending of the spine at the neck and mid-section as compared to the previous year. This will be monitored. 
Figure 12: Changes in posture over 1 year. The prism correction prescribed was $5^{\Delta}$ base down RE and $41 / 4$ base down LE.
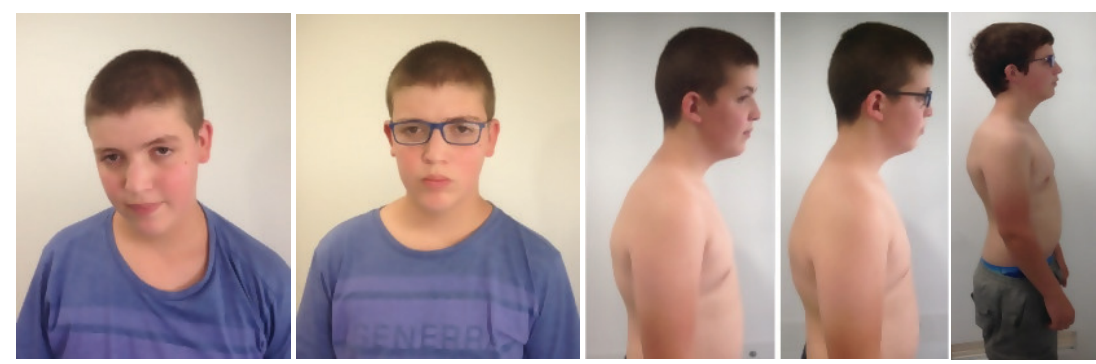

FACIAL ASYMMETRY FROM GROWTH DISPARITY

Asymmetrical development of the face, without involvement of the mastoid process or SCM muscles, can result in one eye being positioned higher than the other.

In most cases, the left eye is higher than the right eye, although the reverse is also encountered. If the activity of the patient involves body movement, without concentrated looking, the maintenance of balance is paramount and the head remains straight. When the patient requires concentrated looking, as opposed to seeing, the head tilts to equalize the heights (Fig. 13). As the head tilts, there is a tendency to create a lateral "S" bend in the posture to balance the centre of gravity. ${ }^{22,23}$ This is seen in Fig. 14. This may also cause a slight cyclo-rotation of the eyes, which may affect the optimal position of the medial and lateral recti, and tracking may become compromised. The use of a small vertical prism, $1 / 4^{\Delta}, 1 / 2^{\Delta}$, repositions the image to the eye, and the head straightens. This re-aligns the spine, reducing stress on the postural muscles and the spinal cartilage, and improves tracking. It is suggested that this adaption is due to the very slow growth-difference in the heights of the eyes. In a child, the height difference is very small. A cortical template of binocular integration is created. As the skull grows, slowly, the relative vertical positioning of the eyes increases slowly, and the head position changes to maintain that original template.

Figure 13: Facial asymmetry due to genetic growth, without involvement of the mastoid process or SCM. During normal "seeing", the head remains vertical (left image for each subject). On "looking", i.e., concentrated viewing, the head tilts to level the eyes (right image for each subject).

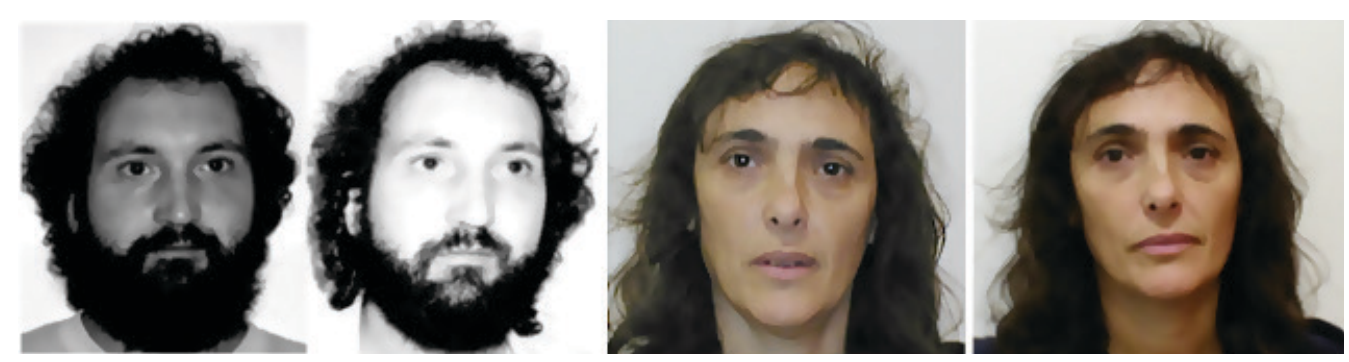

Figure 14: To maintain balance, the spine adapts and an " $\mathrm{S}$ " bend forms.

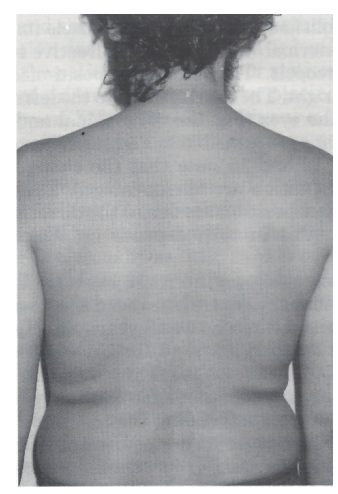


DUANE'S SYNDROME AND LATERAL RECTUS PALSY

The literature mentions the use of prisms to treat horizontal head-tilt, such as in Duane's syndrome, but does not specify whether the prisms prescribed are Differential or Yoked..$^{25}$

Yoked prisms can be used in Duane's syndrome and $6^{\text {th }}$ nerve palsy, where the null point of binocular single vision occurs when the head is turned sideways. This head-turning is both cosmetically disturbing and, during certain monotonous actions such as computer or TV use, as well as driving, can lead to a y-axis twist of the spine and stress on the neck muscles.

Figure 15: Duane's syndrome and 6th Nerve palsy. Use of a horizontal Yoked prism to reduce head-turning.
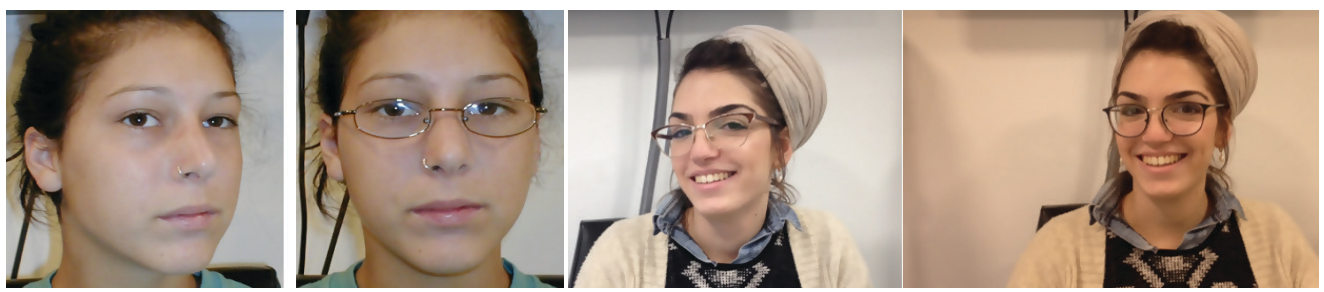

Horizontal Yoked prisms are used to position the head to be as straight as possible. Prisms add weight and thickness, as well as a reduction in visual sharpness. A compromise may be necessary. In Fig. 15, the patient on the left, with Duane's syndrome, is fully corrected. She has minor refractive needs and her glasses only require prisms.

The other patient, with a $6^{\text {th }}$ nerve palsy, required a $12^{\Delta}$ Yoked right horizontal prism combined with a vertical prism to fully correct the posture. Although the head-position was rectified, the first pair of glasses produced, with Yoked $12^{\Delta}$, was rejected by the patient because the optical distortion, weight, and cosmetic appearance were not acceptable. The second pair of Yoked $8^{\Delta}$ was also rejected because there were unacceptable visual distortions. The final (acceptable) prescription was Yoked $6^{\Delta}$ right. With the final prescription, the functionality of daily life was improved, and neck-ache and back-ache were reduced, although not completely alleviated.

The method used to determine the amount of prism required involves a Brock string (Fig. 16). The patient finds the null point without wearing prisms. Yoked prisms are introduced while maintaining the null point, until the patient feels that the body is aligned. Vertical prisms may be included if the patient reports that the two perceived lines of the Brock string are not of the same height.

Figure 16: Use of a Brock string to determine the null point and the amount of Yoked prism required.
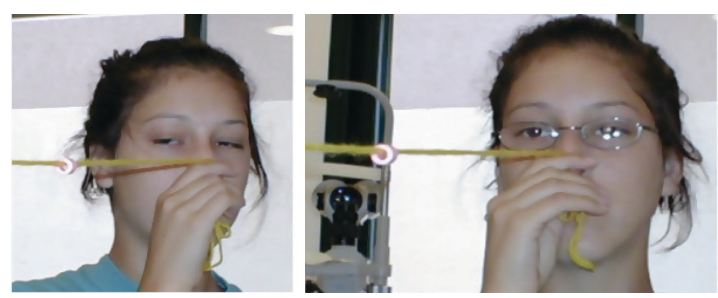

Yoked prisms can also be prescribed to alleviate food spillage from the mouth, and dry mouth in cases of slack-jaw. ${ }^{26}$ Patients with high plus lenses or a frame that does not maintain its position and slips down will be looking through lenses that create a base down prism. This causes the image to appear higher. The patient will raise their head, and possibly their eyes. The jaw tends to drop further and spillage may occur while eating. Dry mouth can also ensue. With the use of a base up Yoked prism in the lenses and with careful choice of the frame, the head position can be maintained and spillage is reduced.

The use of Yoked and Differential prisms to adjust the visual space and spatial localization is independent of the visual needs of the patient, and should not be confused with orthoptic treatment. 


\section{THE AGEING POSTURE}

The leading cause of visits to the emergency departments of hospitals, in an ageing population, is injuries due to falls. ${ }^{27,28}$ The connection between falling in the elderly and vision-related causes has been well established. ${ }^{29,30}$

Vision provides significant input to postural control, as well as information about the size and position of hazards and obstacles. Most multi-focals, and some bifocals, have prism thinning (Yoked prisms) incorporated into their design, for cosmetic reasons and to reduce weight., ${ }^{2,31}$ The Vestibulo-Ocular Reflex (VOR) has been shown to be disrupted by the use of multi-focals. ${ }^{3}$ Adaption is easier in younger patients, but may be problematic in older wearers, who exhibit less muscle tonus. The prescription of single vision correction, as opposed to multifocals, has been recommended. ${ }^{29,32}$ This paper mainly deals with body-based proprioception.

The loss of body strength, muscle mass and tonus as the body ages causes stooping (Fig. 17). The head moves forward and drops. This positions the centre of gravity further forward. As the head droops, there may be difficulty in keeping the eyes raised. This may result in a loss in the upper visual field, and objects positioned further away may be missed. This lessens the ability to pre-empt obstacles. The person tends to lean forward, and may rely on a walking stick to stabilize the body. Older people tend to shuffle their feet when walking, as stepping forward pushes the centre of gravity too far forward, and there is a feeling of insecurity. This is especially difficult when navigating stairs and curbsides.

Figure 17: (a) Head-drooping due to loss of muscle strength in an elderly man. The centre of gravity is moved forward. The patient shuffles and feels insecure. (b) Use of a Yoked down prism raises the spatial localization. The head is raised. The centre of gravity is better aligned. Walking improves.

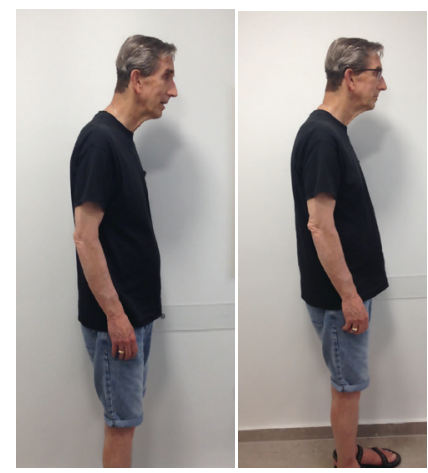

With the addition of a base down Yoked prism, there is a tendency for the patient to lift their head. The centre of gravity is moved backwards. The patient compensates for this by re-positioning their knees and back. The improvement in stability and movement is the result of a combination of factors. There is an increased peripheral awareness. The EOMs operate in a position that has an improved range of flexibility. The peripheral space being processed will increase. This increase in the peripheral signal will also give the incentive to raise the head. This effect has been noted on the Van Orden Star test (VO Star). ${ }^{3}$

When subjects start with Yoked prisms, walking improves, as there is less shuffling and better stepping. The stride length increases. The patient reports feeling more balanced and secure. Going downstairs and stepping off curbs become easier and less hazardous. In some cases, the reliance on a walking stick is reduced.

However, problems have been reported. As seen in Fig. 17, although the head is raised, the posture of the back changes, creating initial discomfort in the leg muscles and lower back. This may improve with time as the muscles strengthen. The adaption to the new posture very much depends on other factors, such as underlying back and knee-joint problems. If there are no major orthopaedic problems, the patient reports rapid adaption to the new arrangement, and a general improvement in mobility and feeling of security.

In a few cases studied, after several months, the glasses were removed to determine if the strengthening of the body muscles was permanent. The posture returned to its original position and the patient experienced the original insecurity in motion. 
PROBLEMS RELATED TO PRESCRIBING PRISMS FOR BALANCING THE POSTURE

It is important to note that no prismatic correction for posture should be incorporated in the prescription if it will compromise the visual needs, or binocular muscle balance, of the patient.

Research by Huang and Ciuffreda suggests that there is a rapid perceptually-driven, sensorimotor adaption as assessed both objectively and subjectively, in response to vertical Yoked prisms. ${ }^{33}$ Prisms above $8^{\Delta}$ can cause a reduction in the quality of vision and should be avoided. Perception through a prism causes expansion of the image on looking towards the base, and compression on looking towards the apex. Erismann and Kohler suggested that patients adjust to this effect. ${ }^{34}$ Furthermore, a horizontal line seen above eye level appears to slope in the direction of the base, while a line below eye level tends to slope upwards. Patients have reported the curvature of straight lines in plano prescriptions with $4^{\Delta}$ prisms. ${ }^{35,36}$ Patients with high prescriptions, when looking away from the optical centre, experience these effects in single vision lenses, but by compensating with head adjustment, vision can be re-aligned with the optical centre. This adjustment is not possible when Yoked prisms are included in the prescription. Sheedy and Parsons, ${ }^{2}$ in their study of patient adaption to Yoked prisms, noted that up to $2^{\Delta}$ Yoked prisms were easily tolerated, while $4^{\Delta}$ prisms were rejected. In my experience, $3^{\Delta}$ vertically Yoked prisms are the limit. In exceptional cases, where a higher prism was necessary, it proved successful. In cases of horizontally Yoked prisms, $5-6^{\Delta}$ were tolerated.

\section{DETERMINING IF PRISMS SHOULD BE PRESCRIBED}

The reaction of patients on receiving Yoked prisms is idiosyncratic. In an attempt to lessen non-tolerance, a further stage is used to determine if a Yoked prism is suitable. This test is totally subjective and cannot easily be verified by objective measurements. The patient is asked to report if they feel that their posture is upright, or if they are leaning forwards or backwards. Since the stability of the patient must also be considered, it is important that the amount of prism is adjusted until the patient reports feeling balanced.

Quaid has suggested that, by observing the patient's walking, the practitioner can gain insight into the suitability of prescribing prisms (personal correspondence).

The patient is requested to walk across a room, and the necessary changes are made to the prismatic prescription, according to the feeling of security and comfort. In the case of an elderly person, the patient is asked to stand and walk, without aids such as a walking stick. If the patient uses a walker (Zimmer frame), the trial is performed with the walker.

There are techniques available for determining if prism prescriptions alter the body balance. These techniques are used mainly for prescribing horizontal prisms. The subject walks along a pressure-sensing mat, and a comparison is made of the differential pressures from each foot as the steps are taken. ${ }^{3}$ This is not suitable for vertical prisms.

The Yoked prism changes the feeling of height, and the patient may need a few days of adjustment. High prescriptions, both plus and minus, are often glazed on the horizontal centre line of the frame, without the optical centres being correctly located. This introduces a Yoked prism. It is important to be aware of this when prescribing and manufacturing glasses.

\section{CONCLUSION}

Although the treatment of orthopaedic anomalies by visual adaption has been pioneered by previous investigators, the integration of these methods in routine optometric practice has frequently been side-lined. In most curricula of optometric education, these methods are rarely stressed. As the scope of practice of the optometric profession changes due to economic pressures, the treatment of orthopaedic conditions by optometric methods should be incorporated into routine practice. $\bullet$

\section{ACKNOWLEDGMENT}

I would like to thank Dr. Patrick Quaid for his immense help in clarifying many of the concepts presented here, and for the time and effort expended in helping prepare this paper.

The patients presented in this paper are from my private practice and have contributed their time to my endeavours. They have all given their permission for their cases to published. I thank them for their help.

Finally, I would like to thank my wife, Ruthie, for her time spent on editing this paper.

CONTACT

email: shapiro.yonatan@gmail.com

Postal Address: POB 1282, Pardes Hanna, Israel, 37000 


\section{REFERENCES}

1. Kaplan M. Vertical yoked prisms. Optometric Extension Program Continuing Education Courses. Santa Ana, CA: Optometric Extension Program Foundation, 1978-1979.

2. Sheedy JE, Parsons SD. Vertical yoked prisms-patient acceptance and postural adjustment. Ophthal Physiol Opt 1987;7(3):255-7. doi. org/10.1111/j.1475-1313.1987.tb00742.

3. Padula WV, Subramanian P, Spurling A, Jennes J. Risk of fall (RoF) intervention by affecting visual egocenter through gait analysis and yoked prisms. NeuroRehabilitation 2015;37(2):305-14. doi: 10.3233/ NRE-151263.

4. Eubank T, Cool T. Improving guided action and perception through use of prisms. J Am Optom Assoc 2001;72(4):217-26.

5. Schmid KL, Beavis SD, Wallace SI, et al. The effect of vertically yoked prisms on binocular vision and accommodation. Optom Vis Sci 2019;96(6):414-23. doi: 10.1097/OPX.0000000000001388

6. Asper L, Leung A, Tran C, Suttle CM, Watt K. The effects of vertical yoked prism on horizontal heterophoria. Optom Vis Sci 2015;92(10):1016-20. doi:10.1097/OPX.0000000000000686.

7. Bansal S, Han E, Ciuffreda KJ. Use of yoked prisms in patients with acquired brain injury: A retrospective analysis. Brain Inj 2014;28(11):1441-6. doi.org/10.3109/02699052.2014.919527

8. Zasler N, Katz D, Ross M. Brain injury medicine: principles and practice. New York, NY: Demos Medical, 2007:523

9. Ashley MJ. Traumatic brain injury, rehabilitation, treatment and case management. Boca Raton, FL: CRC Press, 2010.

10. Suter P, Harvey L. Vision rehabilitation. Boca Raton, FL: CRC Press, 201:209.

11. Padula WV, Argyris S, Ray J. Visual evoked potentials evaluating treatment for post-trauma visions syndrome in patients with traumatic brain injuries. Brain Inj 1994;8(2):125-33.

12. Padula WV, Munitz R, Magrun M, eds. Neuro-visual processing: An integrated model of rehabilitation. Santa Ana, CA: Optometric Extension Program Press, 2012.

13. Padula WV, Nelson CA, Benabib R, Yilmaz C, Krevisky S. Modifying postural adaptation following a CVA through prismatic shift of visuo-spatial egocenter. Brain Inj 2009;23(6):566-76.

14. Velay JL, Roll R, Lennerstrand G, Roll JP. Eye proprioception and visual localization in humans: Influence of ocular dominance and visual context. Vis Res 1994;34(16):2169-76.

15. Rotating tunnel optical illusion. YouTube Web site. https://www. youtube.com/watch?v=hUW6516VJA4. Published May 13, 2017. Accessed November 16, 2019.

16. Quaid P, Hamilton-Wright A. Diagnosing extraocular muscle dysfunction in clinic: comparing computerized Hess Analysis, Park's 3-Step Test and a novel 3-Step Test. Optom Vis Dev 2010;41(3):143-57.

17. Urrets-Zavalía A Jr, Solares-Zamora J, Olmos HR. Anthropological studies on the nature of cyclovertical squint. Br J Ophthalmol 1961 Sep;45(9):578-96.

18. Urrets-Zavalía A Jr. Significance of congenital cyclo-vertical motor defects of the eyes. Br J Ophthalmol 1955 Jan;39(1):11-20.
19. Shapiro IJ. Parallel-testing infinity balance. Instrument and technique for the parallel testing of binocular vision. Optom Vis Sci 1995;72(12):916-23.

20. Shapiro IJ. A new instrument and technique of refraction and binocular balance. The Optician (UK) 1998;215(5643):34-40.

21. Quaid P, Simpson T. Association between reading speed, cycloplegic refractive error, and oculomotor function in reading disabled children versus controls. Graefes Arch Clin Exp Ophthalmol 2013;251:169-87. DOI 10.1007/s00417-012-2135-0

22. Shapiro IJ. Relation between vertical facial asymmetry and postural changes of the spine and ancillary muscles. Optom Vis Sci 1994;71(8):529-38.

23. Shapiro IJ. Examination techniques in cases of vertical facial asymmetry. The Optician (UK) 1993;205(5402):20-7.

24. Major A, Maples WC, Toomey S, DeRosier W, Gahn D. Variables associated with the incidence of infantile esotropia. Optometry 2007;78:534-41

25. Aygit ED, Kocamaz M, Inal A, et al. Management of Duane retraction syndrome with prismatic glasses. Clin Ophthalmol 2017;11:697-700.

26. Shapiro IJ. The use of Yoked prisms to improve the appearance and function of slack-jaw in cases of hypotonia. Optometry Today (UK) 2006;12:38-9.

27. Goldberg EM, McCreedy EM, Gettel CJ, Merchant RC. Slipping through the cracks: A cross-sectional study examining older adult emergency department patient fall history, post-fall treatment and prevention. R I Med J 2017;100(12):18-23.

28. Gelbard R, Inaba K, Okoye OT, et al. Falls in the elderly: a modern look at an old problem. Am J Surg 2014;208(2):249-53.

29. Elliot D. Blurred vision, spectacle correction and falls in older adults. Optom Vis Sci 2014;91(6):593-601.

30. Adams T. Connecting falls to elder vision. Optom Vis Sci 2014;91(6):591-2.

31. Shapiro IJ. The effect of vertical facial asymmetry on the fitting of progressive addition lenses (PAL). Optometry Update (Israel) 1994;1(2):13-5.

32. Haran MJ, Cameron ID, Ivers RQ, et al. Effect on falls of providing single vision distance glasses to multifocal glasses wearers: visible randomised controlled trial. BMJ 2010:340:c2265.

33. Huang MA, Ciuffreda KJ. Short-term adaptation to vertical yoked prisms. Optom Vis Sci 2006;83(4):242-8.

34. Sachse P, Beermann U, Martini M, Maran T, Domeier M, Furtner MR. "The world is upside down” The Innsbruck Goggle Experiments of Theodor Erismann (1883-1961) and Ivo Kohler (1915-1985). Cortex 2017;92:222-32. doi: 10.1016/j.cortex.2017.04.014.

35. Atchison DA, Suheimat M. Theoretical study of refraction effects of plano ophthalmic prisms. Optom Vis Sci 2019;96(1):35-42. doi: 10.1097/OPX.0000000000001321.

36. Atchison DA, Lu J, Yip C, Suheimat M, Schmid KL. Experimental study of refraction effects of nominally plano ophthalmic prisms and magnifying lenses. Optom Vis Sci 2019;96(2):111-6. doi: 10.1097/ OPX.0000000000001334. 